\title{
An Optimized Telecommunication System Topology for Expenditure Minimization and Improved Spectral Utilization Efficiency
}

\author{
Ogunwolu $\mathrm{L}^{1 *}$, Ibidapo-Obe $\mathrm{O}^{2}$ and Adeyemi $\mathrm{T}^{1}$
}

${ }^{1}$ Department of Systems Engineering, Faulty of Engineering, University of Lagos, Nigeria

${ }^{2}$ Vice-Chancellor's Office, Federal University, Ndufu Alike Ikwo, Ebonyi, Nigeria

\begin{abstract}
Capital expenditures (CAPEX) and operational expenditure (OPEX) has always been on the high side if transmission networks are not efficiently planned to minimize these costs. The arrays of antenna often sighted on mast/tower around often post a major risk to the industry as CAPEX increases with increased hardware and signal processing cost. This paper explores the use of point to multipoint (PMP) method of backhauling traffic for cognitive radio network as veritable alternative to existing transmission Point to Point (PTP) Topology in other to minimize the CAPEX and OPEX as well as improve spectra utilization efficiency. The work is focused on small cell deployment for more data penetration in a redundant setup to improve system availability. In the work, the Dijkstra Algorithm for Shortest route analysis was used to prove the advantage of Point to multi point approach in the optimal path planning in a transmission network taking cognizance of path costs as the capital expenditure, path loss, latency, throughput, frequency channel allocation. Furthermore, a spectrum utilization problem was defined, formulated and analyzed. Solution of this model was validated using a simulated setup on a section of Ikorodu ( $6^{\circ} 35^{\prime \prime} .37 \mathrm{~N}$ and $\left.3^{\circ} 31^{\prime \prime} .53 .99 \mathrm{E}\right)$ transmission axis. Results obtained from the analysis show that our model of PMP method of transmitting/backhauling is more capable of improving the spectral efficiency of a wireless telecommunication network and outperforms the PTP topology on all network measures influencing capital and operational expenditures.
\end{abstract}

Keywords: Point to multi-point; Point to point; Shortest route; Network performance measures; Spectrum efficiency

\section{Introduction}

With the rapid growth in wireless industry that gives room for multiple accesses, there is need for channel optimization for more efficient transmission. Spectrum sensing (SpSe) optimization at the physical layer is directly proportional to the class of optimization which reduces the interference in cognitive radio networks.

With increasing number of "data hungry devices" in mobile networks, it is very paramount to initiate an efficient resource control/ allocation technique to maximize the network throughput as well as effective spectral utilization. Transmission optimization technique is employed to maximize effective backhauling of data traffic from one node to another. This work lays emphasis on cooperation among several multiple radios based on distributed detection technique.

It is aimed at establishing the overall spectrum utilization enhancement using improved system Topology. The specific objectives of this work are:

- To determine the effectiveness of Topology that makes use of point to multipoint (PMP) method of backhauling.

- To investigate the preference of the Point to Multipoint (PMP) topology over the Point to Point (PTP) topology on the basis of minimisation of Capital Expenditure (CAPEX)

- To investigate possible preference of the PMP topology over the PTP topology on the basis of Operating Cost (OPEX) influencing network performance measures

- To investigate the performance of both the PMP and the PTP topologies on the basis of spectrum utilization.

\section{On spectrum utilization}

Spectrum is a collection of feasible wavelength of electromagnetic radiation.

Economists and radio engineers are always at loggerhead over the allocation system of spectrum which is widely believed to be wasteful with little or no emphasis on efficiency. They both believe the present system of spectrum utilization is faulty and needs redefinition. While economists argue on market capitalization basis, the engineers define spectrum utilization on what is obtainable (Figures 1 and 2).

The license acquired for spectrum allocation could only be used for the purpose approved for. Efficient and optimal allocation of spectrum by relevant agencies in order to prevent interference is of utmost importance.

\section{On demand for spectrum}

The astronomical growth in number of telecommunication providers providing wireless access has made the demand for spectrum more competitive. With more demand for data rather than voice, the broadband data access now requires more bandwidth than the traditional voice requirement for bandwidth.

With the industry profit now centred on broadband data access, there are more reasons to use the ever scarce spectrum allocated effectively and efficiently.

\section{On spectrum sensing}

Factors to be considered in deployment include topology, frequency and licensing (Figure 3).

*Corresponding author: Ladi Ogunwolu, Department of Systems Engineering, Faulty of Engineering, University of Lagos, Nigeria, Tel: 08023028914; E-mail: fogunwolu@unilag.edu.ng

Received December 31, 2014; Accepted June 29, 2015; Published July 07, 2015

Citation: Ogunwolu L, Ibidapo-Obe O, Adeyemi T (2015) An Optimized Telecommunication System Topology for Expenditure Minimization and Improved Spectral Utilization Efficiency. J Telecommun Syst Manage 4: 119. doi:10.4172/2167-0919.1000119

Copyright: @ 2015 Ogunwolu L, et al. This is an open-access article distributed under the terms of the Creative Commons Attribution License, which permits unrestricted use, distribution, and reproduction in any medium, provided the original author and source are credited. 


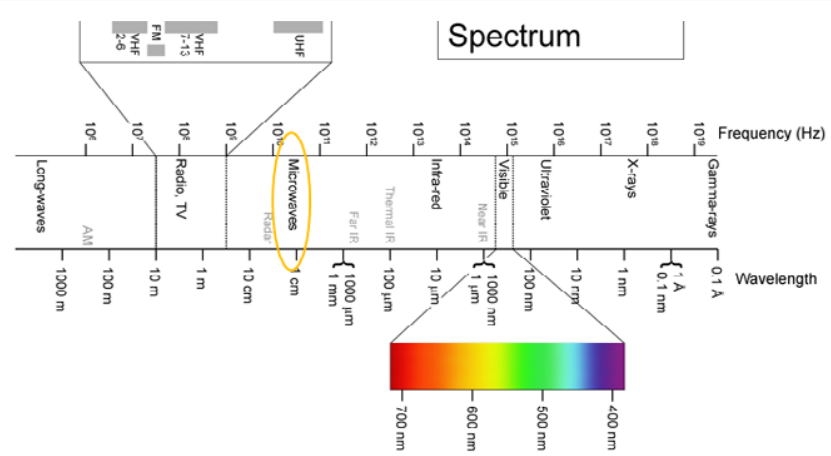

Figure 1: Electromagnetic spectrum.

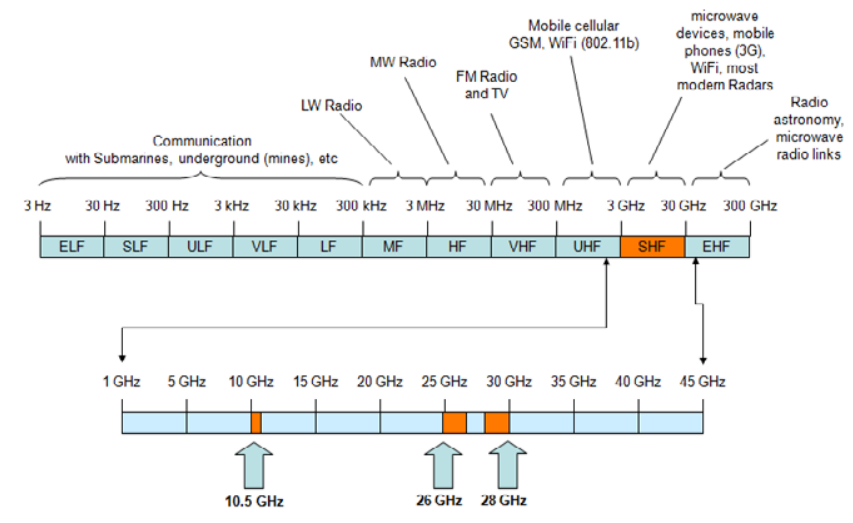

Figure 2: The microwave spectrum.

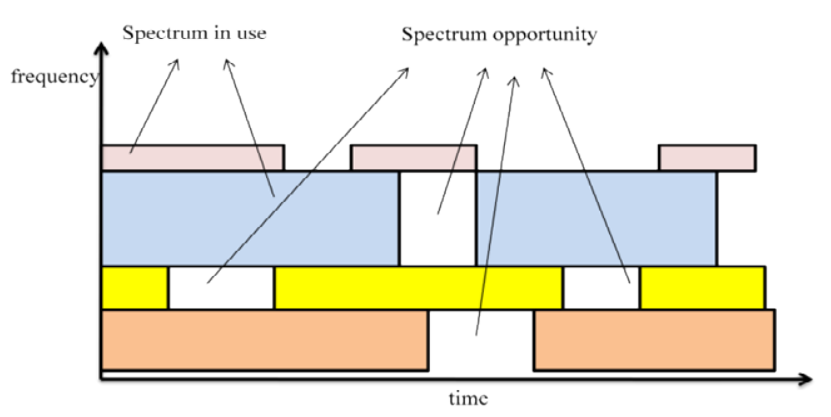

Figure 3: Spectrum sensing.

The time it takes to sense the transmission channels, the signal strength, and the priority value on the signal are the most paramount parameters that determine the performance of the spectrum sensing.

Spectrum sensing determines the available channel holes for licensed users.

\section{On cognitive radio}

The concept of Cognitive Radio was first addressed by Mitola and Maguire [1] in their work. The subject matter has been a subject extensive research and technical discussions since Mitola and Maguire first used it.

Cognitive radios enable collocation with other secondary networks competing for the same spectral resources in realizing an effective protocol for real-time radio spectrum rental.

\section{The current approach-point to point (PTP) backhauling}

The PTP system is currently the most widely used method of backhauling traffic where the assigned pairs of radio frequency channels are configured onto pairs of transceivers. This significant wastage of spectrum prompted several researchers on series of researches in optimizing the spectrum allocated. The approach of deployment does not have channel arbitration in the form of a medium access control (MAC) scheme required because only a single pair of transceivers is allocated to a channel (Figure 4).

This type of system may be conceived of as a circuit-switched network Topology, where the circuit is the statically-allocated radio frequency (RF) channel.

\section{Point to multi-point (PMP) deployment}

In contrast to PTP, for a point-to-multipoint (PMP) system channel arbitration of some kind is a necessity. In PMP, the central station broadcasts downstream traffic with some kind of additional identifying addressing field. All of the remote terminal devices (RTDs) are able to receive and decode at least the addressing fields and thus can discriminate traffic which is destined for them from traffic destined for other RTDs. A form of medium access control is used on the upstream direction whereby an RTD can indicate that it has data to transmit and is granted access to the channel while transmission by other RTDs is suppressed. The upstream and downstream directions are thus multiplexed, and the usual multiplexing methodologies may be employed; some form of dynamic time division multiple-access is common. A PMP system embodies a packet-switched Topology (Figure 5).

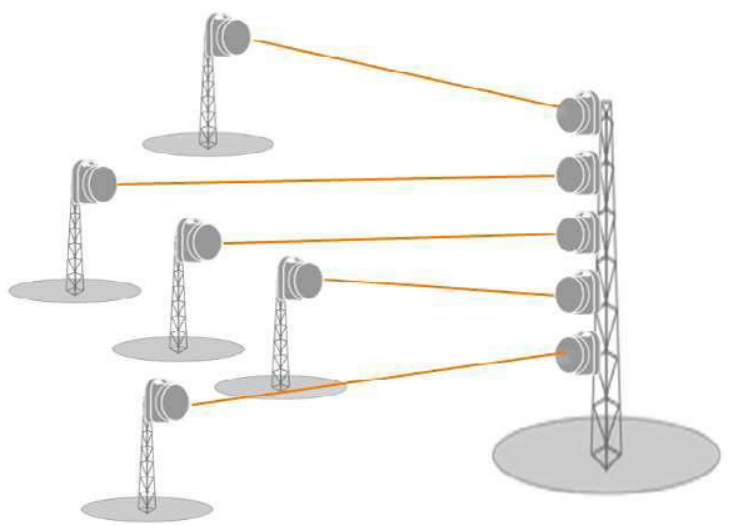

Figure 4: Point-to-point star topology.

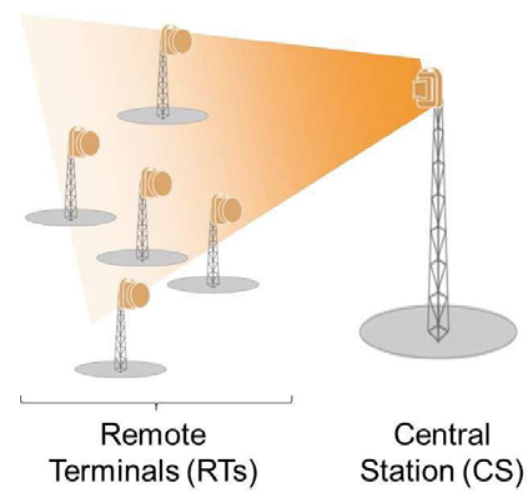

Figure 5: Point-to-multipoint topology. 


\section{Similar research work}

European Space Agency Telecommunications and Integrated Application (EIA-TIA) Directorate is currently conducting research on "Next Generation Waveform for Improved Spectral Efficiency". The agency that is saddled with the responsibility of coordinating, shaping and supporting improvement in satellite telecommunications and for the advancement of applications that involve the collective use of spacebased telecommunications, earth observation and navigation systems has invested in and facilitated numerous research and development works [2].

The proposed EIA-TIA design will enhance waveforms to be used by the forward and return links of next generation broadband and narrowband satellite networks, together with the associated estimates in terms of system performance improvements and high level complexity. It will also take into consideration the investigation of the air interface enhancements, definition and assessment of the new air interface, system performance assessment and technology roadmap to reduce the spectral efficiency.

The research is still ongoing with no result obtained till date.

\section{Significance of research work}

This work will

- Enhance the green environment by reducing the harmful effects of close proximity of array of antennas to human body,

- $\quad$ Reduce the CAPEX and OPEX

- Result in the effective utilization of the scarce spectrum, and

- $\quad$ Reduce expended energy as a result of reduced equipment usage.

The rest of this paper is organized as follows. In Section 2, related research to the subject matter is summarily reviewed. In Section 3 , the methods used in analysis of the test network are explained while the analyses of the network problem are exhibited in Section 4. Finally, in Section 5 conclusions are drawn on the work and possible future extensions highlighted.

\section{Related Work}

The backhauling of traffic due to rapid growth of wireless network is now being developed to behave as an intelligent transport system (ITS) making use of opportunistic utilization of spectrum sensing to intelligently discover the under-utilized frequency bands without causing any constructive or destructive interference. The spectrum sensing aids the dynamic usage of spectrum efficiently with minimal interference to the legacy network [3].

ZhiQuan et al. [4] in their paper conducted empirical analysis on Wideband Spectrum Sensing in Cognitive Radio Networks. Though this subject of discussion has limited number of literature for discussion, they were able to develop the optimization problem based on the development of joint formulated problems to reduce the interference by evaluating the "unapproached" convexity in the open non convex problems under field conditions. Himal et al. [5] described Cognitive Radio as intelligent method of backhauling of radio frequency with coherent adaptation to transmission parameters in a cohesive electromagnetic environment to improve the overall spectral efficiency (Figure 6).

Rui Zhang et al. [6] further described the cognitive radio operation model as Interweave, Overlay, and Underlay. They discussed that interweave, also known as the opportunistic spectrum access (OSA),

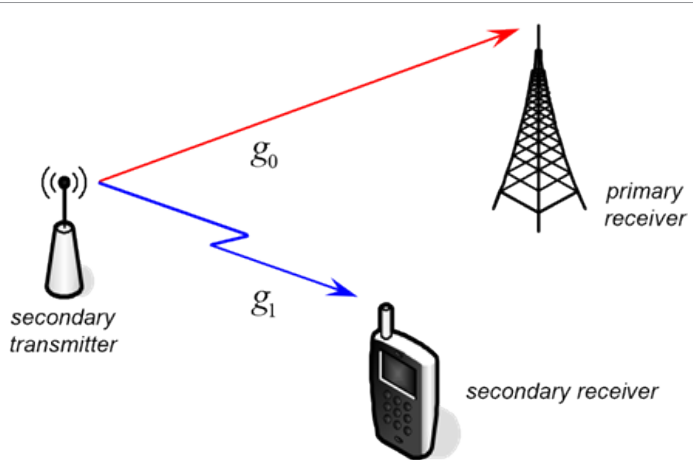

Figure 6: Shared spectrum usage between primary and secondary users.

exists where the Cognitive radio disseminates power over the unique spectrum to the existing primary radio when the primary radio is at zero transmission. The overlay and underlay methods permit the primary radios to transmit concurrently on the same frequency platform.

The design of clustered Topology for cooperative spectrum sensing with optimization of number of clusters was discussed by Katre Apurva et al. [7] in their publication. They described the cognitive radio environment as an entity which consists of several multiple secondary users and primary users with the opportunity of healthy colocation of cognitive radios within primary and secondary networks.

Katre Apurva et al. [7] concluded that cooperative spectrum sensing enhances the spectrum utilization. Optimization of number of clusters and nodes in the cluster effectively reduces the power consumption and also increases the probability of signal detection due to weighted cluster algorithm which in turn increases the efficiency of the algorithm.

The spread spectrum based wireless technology is currently being designed to support broadband moving of multimedia data traffic as against the traditional technology.

The interoperability of licensed users and unlicensed users has made the Cognitive radio the next generation wireless method. The licensed users have sole right to the ownership of the spectrum when needed while the unlicensed users have to scout for the availability of the unused spectrum. The unlicensed users or secondary users employ the strategy of spectrum sensing to discover the available spectrum.

Moslem Rashidi et al. [8] define the secondary utility function $U_{s}(p(t), t)$ as:

$$
U_{s}(p(t), t)=\max \{I(p(t), t), S(p(t), t), T(p(t), t)\}
$$

Where $I(p(t), t), S(p(t), t)$, and $T(p(t), t)$ are the secondary users' maximum expected utilities for taking the action of staying idle, carry out spectrum sensing or make data transmission respectively.

This work proposes a topological redesign for the problem of backhauling traffic from various multi nodes to a central point (Access Point, AP) to increase spectral efficiency, reduce hardware/ signal processing cost and enhance effective utilization of the spectrum.Performance problems experienced by the end users will be considerably reduced with the implementation of point to multipoint Topologyin wirelessly communication networks occasioned by transferring both the energy and information from one point to several points. The AP broadcasts via the downstream channel while the users return the information/data via the upstream channel.

Several discussions have made emphasis on spectrum sensing as an effective means of spectrum utilization with little or no emphasis to the 
network topology as one of the major issues in spectrum management. Several studies on both technical and policy issues related to dynamic (and open) spectrum access management and utilization argue on segmenting the users into primary and secondary users with emphasis on quoted-weighted sharing as an avenue to manage the users from excessive overbooking.

Santosh Kumar Singh et al. [9] proposed the use of genetic algorithm for spectrum management of cognitive radio. Genetic algorithm is known to have capability to solve complicated problems like non-deterministic problems and has found applications in the evolution of simple programs like evolution of images, music and video games.

\section{Methods of Analysis}

Strict utilization of allocated spectrum without any interference is assumed in this work. Base station (BS) performance is classified on the effective throughput and availability while overall performance is analyzed using shortest route analysis.

The utilization of the spectrum by the BS is defined as $\zeta$ of the $j^{\text {th }}$ spectrum band where

$$
\zeta=\Sigma_{j}\left(B_{i j 2}-B_{i j 1}\right)
$$

assuming no interference from neighborhood Radio Frequency sources. Here, $\left(B_{i j 2}-B_{i j 1}\right)$ is the range between the lower and upper bounds of the $\mathrm{j}^{\text {th }}$ spectrum band. In a network, the optimal spectrum utilization is the maximum total utility over all Base Stations.

The Topology efficiency $\eta_{s y s}$ is defined as probability efficiency metric for the remote terminals device (RTD) or users which depend on the overall efficiency and the total network capacity.

Denote sets of RTDs by $\mathrm{N}=\{1,2,3,4 \ldots, N\}_{s}$

We analyze an area for spectrum utilization with a frequency division model for spectrum sharing where the users co-exist on a channel. It is also assumed that each RTD exists on a separate transmitter-receiver pair registered on the Access Point with $\mathrm{b} \mathrm{Hz}$ bandwidth and channel gain $g_{r t d}$.

The channel rate $\mathrm{R}$ is defined as:

$$
R=b \log \left(1+\frac{g_{r t d} P}{N_{o}}\right)
$$

It is also assumed that at the point of spectrum allocation, the users and sellers have probabilistic information $P$ about the channel gain $g_{r t d}$ with channel density $\Omega$.

The expected rate with respect to $\mathrm{b} \mathrm{Hz}$ of bandwidth is given as $\varphi$.

$$
\text { Where } \varphi(b)=\int b \log \left(1+\frac{g_{r t d} P}{N_{o}}\right) \Omega\left(g_{r t d}\right) d\left(g_{r t d}\right)
$$

We assumed equation 4 is well defined for all $0<\mathrm{b}>\mathrm{C}$

Where $\mathrm{C}$ is the total number of channels accruing to the operator

From equation 4

$$
\begin{gathered}
\varphi^{t}(b)=\int\left[\log \left(1+\frac{g_{r t d}}{N_{o} b}\right)-\left(g_{\frac{r t d}{N_{o} b+g_{r t d} \Omega}}\right)\right] \Omega\left(g_{r t d}\right) d\left(g_{r t d}\right) \\
u(b)=\int\left[-\left(\frac{g_{r t d} P}{\left(g_{r t d} P N_{o} b\right) b}\right)+\left(g_{\frac{r t d}{\left(N_{o} b g_{r d d}\right)}}\right)\right] \Omega\left(g_{r t d}\right) d\left(g_{r t d}\right)
\end{gathered}
$$

$$
=\int\left[-\left(\frac{\left(g_{r t d} P\right)^{2}}{\left(g_{r t d} P+N_{o} b\right) b}\right)\right] \Omega\left(g_{r t d}\right) d\left(g_{r t d}\right) \leq 0
$$

Equation 6 proves the concavity of $\varphi(b)$, also $\varphi^{\dot{e}}(0)=+\infty$ and $\lim _{b \rightarrow \infty} \varphi^{t}(b)=0$ for PMP or Cognitive radio where users coexist on a unique channel.

This shows that $\varphi^{t}(b)$ is a non-increasing function which implies $\varphi^{t}(b)>0$ for $b \geq 0$.

\section{The shortest network route problem}

Shortest path algorithms can be used to determine the best link path approach between a source and destination in a transportation or backhauling network problem. Many industrial problems can be modeled and solved as networks using nodes connected by links (arcs) provided the link costs can be estimated (Figure 7).

Networks definition: In our problem setting, the Base Stations (BS) constitutes the nodes while the arcs are the transmission/radio links between the nodes. Thus, the transmission Topology can be fully characterized as:

$$
\Omega_{\mathrm{sys}}=(\mathrm{N}, \mathrm{A})
$$

Where,

$\mathrm{N}=$ set of nodes $=\{1,2, \ldots \ldots, \mathrm{n}\}$ and

$\mathrm{A}=$ set of corresponding transmission links $=\left\{\ell_{\mathrm{ij}}\right\}$, provided $i \neq j$ and transmission link between the $i^{\text {th }}$ and the $\mathrm{j}^{\text {th }}$ nodes exists, $i, j=1,2$, ...., $n$. It is a directed network.

In our case, the link costs, $c_{i j}$, are represented as calculated or estimated unit-paritized values of capital cost, path loss, availability, latency and channel frequency allocation on each link $l_{i j} i, j=1,2, \ldots, n$ These link costs are obtained from parameterized functions developed for this purpose or obtained from literature.

On a general basis, let,

$x_{i j}=$ amount of flow on arc $(i, j)$

$c_{i j}=$ link cost on $\operatorname{arc}(i, j)$

The objective function is to minimize the total cost

$$
\text { Minimize } z=\sum_{\begin{array}{c}
\text { all defined } \\
\text { arcs }(i, j)
\end{array}} c_{i j} x_{i j}
$$

This is solved using the Dijkstra's algorithm. This algorithm is more appropriate and economical for use in this case since it computes the shortest route from a source node to any other node in the network as opposed to algorithms like Floyd's which computes for any pair of nodes in the network[10].


Figure 7: Point-to-point topology. 
The test network: The selected test arena is the Ikorodu municipality and environ. The Base Transmission Station (BTS) for the test network is selected as located in Ikorodu on Latitude $6^{\circ} 35^{\prime \prime} .37 \mathrm{~N}$ and Longitude $3^{\circ} 31^{\prime \prime} .53 .99$ E. $20 \mathrm{~m}$ above the sea level. Seven nodes were chosen on the terrain to accommodate all traffic from Ikorodu town taking the complexity of the terrain into consideration. The BTS coveragewas configured with a beam-width of $90^{\circ}$-sector so that the AP can give $90^{\circ}$ coverage to all seven nodes (Figures 8 and 9).

The relative distances, in kilometers, between the selected nodes on the chosen network are obtained from the Google Map tool and are as depicted on the network configuration (Figure 9). Node 1 represents the base station. The network is a directed network. The test network nodes were chosen in such a way to be able to illustrate the two alternate topologies (PTP and PMP) under discussion. For example, all other nodes are directly connected to node 1 (the base station), which is the PMP feature, while all other nodes, except node 4, are connected through other node(s) to node 1 (the base station) for transmission and or backhauling, a feature of the PTP Topology.

PMP versus PTP: The decision on method of backhauling depends on Network planners who will always take into cognizance the availability, fade margin, topography, etc. before arriving at the final decision. The PTP method is usually used when traffic is smooth and uncongested especially in the middle of backhaul.

The PMP method encourages on-air traffic aggregation for a bursty/ data dominated network especially when the spectrum is congested and expensive making it better than the PTP as it improves the efficient usage of spectrum.

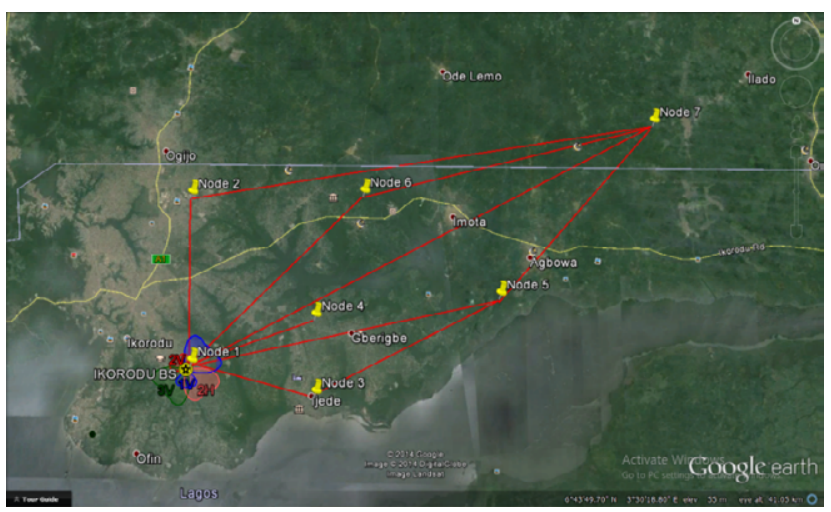

Figure 8: The test network (Google map view).

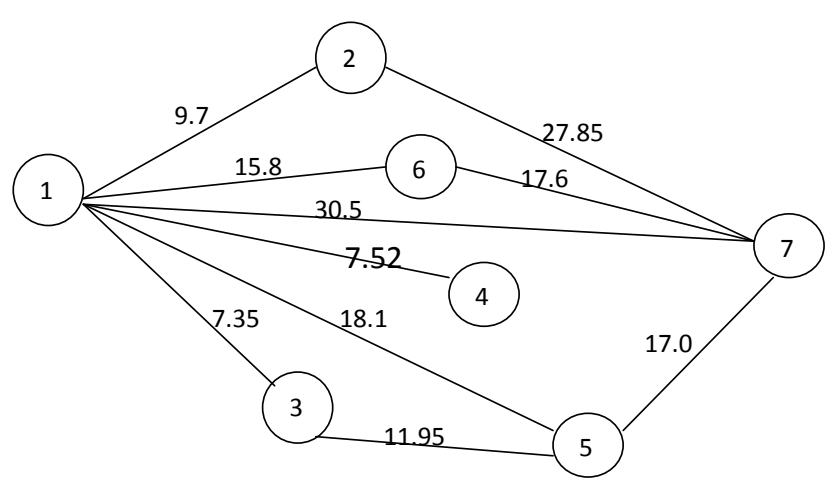

Figure 9: Network configuration.

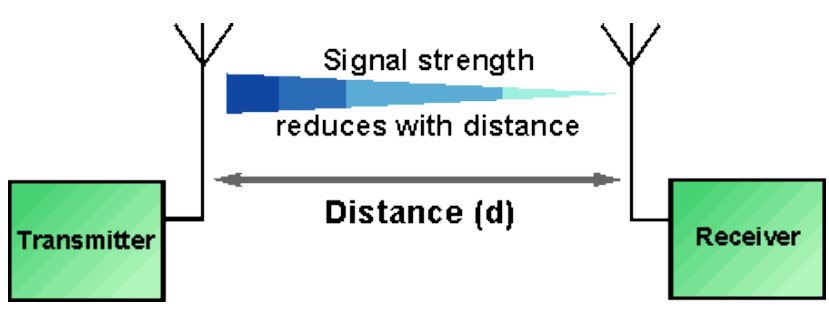

Figure 10: Free Space Path Loss analysis.

Capital expenditure (CAPEX) for PTP and PMP: The Capital Cost or Capital Expenditure (CAPEX) depends on a lot of things which center on investment on the equipment. In this work, the equipment usages on PTP and PMP to backhaul traffic to the nodes' Outdoor unit setup (ODU) are compared. To backhaul traffic from n nodes, using the Point to Point (PTP) Topology, the required number of microwave radios to be used is $2 \mathrm{n}$. For the Point to Multi-Point (PMP) setup the number required is $n+2$ or $n+1$ for the case of one redundant setup at the back endor non-redundant setup respectively. It is obvious that $2 n>n+2>n+1$ for any value $\in Z$ where $Z$ is real number. This translates to reduction of capital expenditure (CAPEX) in favor of the PMP setup.

Free space path loss (FSPL): The reduction in signal strength as electromagnetic wave travels over a distance where we assumed no obstacles that can cause refraction, diffraction, reflection, etc. is known as FSPL (Figure 10).

$$
\mathrm{FSPL}=\left(\frac{4 \pi \mathrm{d}}{\mathrm{c}}\right)^{2}
$$

Where: FSPL is the free space path loss

$\mathrm{d}=$ distance of the receiver from the transmitter

(meters) (Figure 10).

$\mathrm{c}=$ speed of light in a vacuum (meters per second)

Signal frequency, $\mathrm{f}$, is inversely proportional to the square of the distance from the transmitter to the receiver, $d . f \propto \frac{1}{d^{2}}$

The Free Space Path Loss (FSPL) is also proportional to distance, $\mathrm{d}$.

$$
F S P L=20 \log _{10}(d)+20 \log _{10} f+32.44
$$

For a constant signalfrequency, $\mathrm{f}$, (in Giga hertz), the last two terms can be regarded as a constant. Thus,

$$
\begin{aligned}
& F S P L=20 \log _{10}(d)+\mathrm{K} \\
& \text { Where } \mathrm{K}=20 \log _{10} \mathrm{f}+32.44
\end{aligned}
$$

In effect, based on (10) and (11) or (11) and (12) where $\mathrm{K}$ is a constant, the distance between the receiver and the transmitter, $d$, can be taken as a measure of free space path loss (FSPL) in lieu of FSPL in (11). This is the measure used in this work.

Hence, Overall Network efficiency is much better with direct connection to the nodes from the Access Point which the PMP setup affords.

Availability: Availability in this context is the percentage ratio of the difference in measured time and the unavailable seconds (UAS). When there are intermediate receivers enroute the transmitter, availability of the destination receiver will be dependent on the availability of the intermediate receivers. The Unavailable Seconds 
Citation: Ogunwolu L, Ibidapo-Obe O, Adeyemi T (2015) An Optimized Telecommunication System Topology for Expenditure Minimization and Improved Spectral Utilization Efficiency. J Telecommun Syst Manage 4: 119. doi:10.4172/2167-0919.1000119

Page 6 of 9

(UAS) of the destination receiver is dependent on the ripple effects of UAS of all the intermediate receivers (Figure 11).

The availability of a node for this work was computed using network planning tools. Availability can be computed, for example, for all the nodes for a $10 \mathrm{GHz}$ Access Point (AP) broadcasting to all the nodes. The availability will be the same for all the nodes connecting directly to the main node while reduction in number of nines $(9 \mathrm{~s})$ is experienced with the connecting nodes depending on the intermediate node(s).

With the direct connection to the remote nodes, the number of point of failures is reduced compared to interconnecting through nodes.

Throughput, latency and back to back tests: Throughput, latency and back to back simulation tests were simulated over the entire test network for comparisons of the PTP and the PMP topology over a range of frame sizes from 64 octets to 1518 octets $(64,128,256,512$, 1024 and 1518 octets). The three measures of system performance directly or indirectly affect the operation expenditure (OPEX).

Throughput is the amount of data successfully delivered per unit time. Throughput is controlled by available bandwidth, as well as the available signal-to-noise ratio and hardware limitations.

In a network, latency, a synonym for delay, is an expression of how much time it takes for a packet of data to get from one designated point to another.

The back-to-back value is the number of frames in the longest burst that can be transmitted without the loss of any frames.

System Operators want to maximize throughput to maximize revenues, while users of a system want low latencies so they don't waste their time.

A laboratory simulation test network was set up to determine throughput across both the PTP and the PMP networks.

The following are the highlights of the test simulated in the laboratory for the topologies under discussion.

- $\quad$ Traffic at the smallest frame size is sent across the network for a fixed time period.

- If the number of frames received is the same as the number of frames transmitted, data rate is increased and the test is re-run.

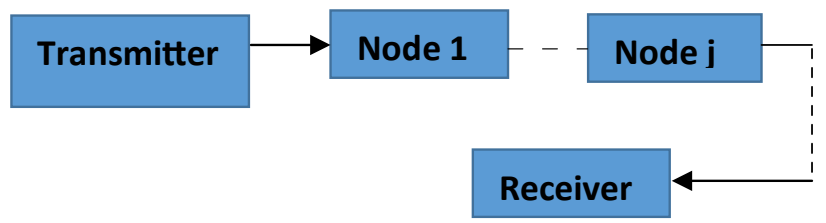

(i) Connection through intermediate nodes

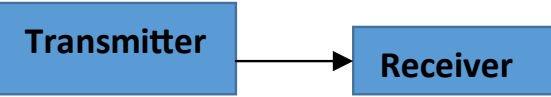

(ii) Direct connection

Figure 11: Availability in series

\begin{tabular}{|c|c|c|}
\hline Number of Nodes & PMP Channel Usage & PTP Channel Usage \\
\hline $\mathbf{1}$ & 1 & \\
\hline $\mathbf{2}$ & 1 & 1 \\
\hline $\mathbf{3}$ & 1 & 2 \\
\hline $\mathbf{4}$ & 1 & 3 \\
\hline $\mathbf{5}$ & 1 & 4 \\
\hline $\mathbf{6}$ & 1 & 5 \\
\hline $\mathbf{7}$ & 1 & 5 \\
\hline
\end{tabular}

Table 1: Channel usage.

- If the number of frames received is less than the number of frames transmitted, data rate is reduced and the test is re-run.

- Once the maximum data rate has been determined, the next frame size is used and the process repeated

RFC 2544 states that the test duration trial length MUST be at least 2 seconds and SHOULD be repeated at least 50 times with the average of the recorded values being reported.

Inference drawn from the results show that PMP backhaul is much more efficient than the PTP method of backhauling.

Frequency channel allocation: The use of frequency division duplex (FDD) system that enables the radio channels to be used simultaneously by all radios is assumed for PMP Topology. This implies in our analysis that single channel information is enough to backhaul the traffic to the central node as they are all within the coverage of one AP.

In the laboratory setup, the table below is the frequency channels used for the test carried out for both PMP and PTP.

To backhaul traffic for four nodes, for example, on the test network, a single channel is required for PMP so far the nodes fall within the beam width of the Access Point while for PTP at least three different channels are required to minimize interference (Table 1).

The result shows minimal channel usage in PMP as against the PTP method of backhauling as downstream channel and upstream channel are the same for PMP nodes. This obviously will translate to lesser operation expenditure (OPEX) for the PMP setup compared to the PTP setup.

Spectrum utilization: The spectrum utilization $U$, is the product of the frequency bandwidth, the geometric (geographic) space, and the time denied to other potential users:

$\mathrm{U}=$ B. S. T

where,

$\mathrm{B}=$ frequency bandwidth

$\mathrm{S}=$ geometric space (usually area) and

$\mathrm{T}=$ time.

A frequency bandwidth of $7000 \mathrm{Mhz}$ is assumed and the geometric space used is that of Ikorodu, an area in the suburb of Lagos, Nigeria.

Spectrum utilization efficiency (SUE), of a radio communication system, can be expressed by:

$$
S U E=\frac{M}{U}=\frac{M}{B \cdot S \cdot T}
$$

where:

$M$ is the amount of information transferred over a distance. 
Citation: Ogunwolu L, Ibidapo-Obe O, Adeyemi T (2015) An Optimized Telecommunication System Topology for Expenditure Minimization and Improved Spectral Utilization Efficiency. J Telecommun Syst Manage 4: 119. doi:10.4172/2167-0919.1000119

In this work, Relative spectrum efficiency (RSE) is defined to compare the spectrum utilization of the two topological systems (PTP and PMP) modes of backhauling when the radio is configured with the same Ethernet circuit. It is defined as the ratio of two spectrum utilization efficiencies.

$$
R S E=\frac{S U E_{p m p}}{S U E_{p t p}}
$$

Where,

$\mathrm{SUE}_{\mathrm{pmp}}=\mathrm{SUE}$ of the PMP system

$\mathrm{SUE}_{\mathrm{ptp}}=\mathrm{SUE}$ of the PTP system

The RSE value is much easier to compute than the SUEs for the individual system and then using them for comparison. The two modes are assumed to provide the same service of backhauling. As a ratio, the common factors in the two measures of utilization efficiency cancel out and the ratio eventually can be reduced to the quotient of number of frequency channels allocated for the PMP and PTP, given by the expression,

$$
R S E=\frac{S U E_{p m p}}{S U E_{p t p}} \equiv \frac{\text { Number Channel allocations for PMP }}{\text { Number Channel allocations for PTP }}
$$

To backhaul traffic for 7 nodes, for example, five different channels are required for PTP mode compared to a single channel required for PMP mode of backhauling.

Hence, equation 16, for that case, becomes

$$
R S E=\frac{S U E_{p m p}}{S U E_{p t p}}=\frac{1}{5}
$$

This translates to the deduction that the spectral utilization efficiency of the PTP system to backhaul traffic over seven nodes is just one-fifth (20\%) of the spectral utilization efficiency of the PMP system. Again, this is another guarantee for lesser operation expenditure (OPEX) for the PMP topology.

\section{Results and Analysis \\ On CAPEX and OPEX}

Since the capital expenditure is a function of the number of components used and the latter is in turn proportional to the number of nodes in the network. The number of components used in lieu of the actual capital expenditure is higher for the PTP Topology than for the PMP Topology for the test network as seen in Table 2.

For example at Node 5, four outdoor units (ODU) are required for the backhauling to node 1 through Node 2 (PTP Mode), while for PMP mode, a single ODU is needed to backhaul to the access point (Table 2 and Figure 12).

\begin{tabular}{|c|c|c|}
\hline NODE & PMP ODU Components Used & PTP ODU Components Used \\
\hline $\mathbf{1}$ & - & - \\
\hline $\mathbf{2}$ & 1 & 2 \\
\hline $\mathbf{3}$ & 1 & 2 \\
\hline $\mathbf{4}$ & 1 & 2 \\
\hline $\mathbf{5}$ & 1 & 4 \\
\hline $\mathbf{6}$ & 1 & 2 \\
\hline $\mathbf{7}$ & 1 & 4 \\
\hline
\end{tabular}

Table 2: PMP/PTP ODU usage.

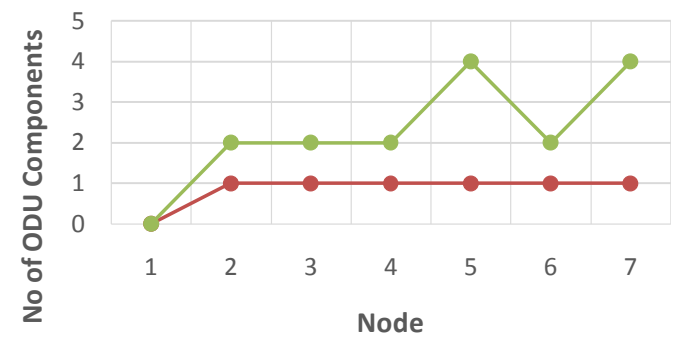

-P PMP Topology —PTP Topology

Figure 12: PTP and PMP ODU usage.

\begin{tabular}{|l|l|l|l|l|l|l|l|}
\hline \multicolumn{7}{|l|}{ Nodes } & \multicolumn{9}{l|}{} \\
\hline Path(T) Km & $\mathbf{1}$ & $\mathbf{2}$ & $\mathbf{3}$ & $\mathbf{4}$ & $\mathbf{5}$ & $\mathbf{6}$ & $\mathbf{7}$ \\
\hline 1 & ---- & 9.7 & 7.35 & 7.52 & 18.1 & 15.8 & 30.5 \\
\hline 2 & 9.7 & ----- & & & & & 27.8 \\
\hline 3 & 7.35 & & ----- & & 11.9 & & \\
\hline 4 & 7.52 & & & ---- & & & \\
\hline 5 & 18.1 & & & & ---- & & 17.0 \\
\hline 6 & 15.8 & 26 & & & & ---- & 17.6 \\
\hline 7 & 30.5 & 27.8 & & & 17.0 & 17.6 & ---- \\
\hline
\end{tabular}

Table 3: Minimum path analysis on the test network.

\begin{tabular}{|l|c|}
\hline Path & Distance (km) \\
\hline $\mathbf{1} \rightarrow \mathbf{7}$ & 30.5 \\
\hline $\mathbf{1} \rightarrow \mathbf{2} \rightarrow \mathbf{7}$ & $37.5(9.7+27.8)$ \\
\hline $\mathbf{1} \rightarrow \mathbf{6} \rightarrow \mathbf{7}$ & $33.4(15.8+17.6)$ \\
\hline $\mathbf{1} \rightarrow \mathbf{5} \rightarrow \mathbf{7}$ & $35.1(18.1+17.0)$ \\
\hline $\mathbf{1} \rightarrow \mathbf{3} \rightarrow \mathbf{5} \rightarrow \mathbf{7}$ & $36.25(7.35+11.9+17.0)$ \\
\hline
\end{tabular}

Table 4: Path analysis from node 1 to node 7.

\begin{tabular}{|c|c|c|}
\hline Component & Availability & Downtime \\
\hline Node 2 & $99 \%$ (2-nines) & 3.65 days/year \\
\hline Node 1 & $99.99 \%$ (4-nines) & 52 minutes/year \\
\hline 1 and 2 Combined (Series) & $98.99 \%$ & 3.69 days/year \\
\hline
\end{tabular}

Table 5: Availability for traffic haul from nodes 1 to 2 .

\section{On free space path loss}

From Section 3.1.4, the free space path loss is shown to be a function of and proportionate to the distance, $\mathrm{d}$, between the transmitter and the receiver. Hence, the Dijkstra's Algorithm can be used to determine the minimum distance path between the transmitter and each of the nodes in the PTP system or Topology for the test network. The distance, d, for each of the nodes in the PMP Topology is the lesser, being direct connections between transmitter and receiver in each case (Table 3).

The Table 3 shows the distance between the nodes. While the distance travelled going to node 7 from 1 is $30.5 \mathrm{~km}$, to reach the same node through node 2 will be $37.5 \mathrm{~km}$, resulting in additional $7 \mathrm{~km}$. By implication, the free space path loss by direct link between node 1 (the Base Station) and node 7 will result in minimum free space loss. The same argument goes for the other nodes in the network and thus the PMP topology eventually proves better that the PTP (Table 4).

\section{On availability}

Availability and resulting downtime from one node to another depends on the path and the number of intermediate nodes in between. For example using network planning tools as earlier explained in 
Citation: Ogunwolu L, Ibidapo-Obe O, Adeyemi T (2015) An Optimized Telecommunication System Topology for Expenditure Minimization and Improved Spectral Utilization Efficiency. J Telecommun Syst Manage 4: 119. doi:10.4172/2167-0919.1000119

Section 3.1.5, the availabilities and the resulting downtimes of nodes 1 and node as well as what obtains for traffic haul from 1 to 2 is depicted in Table 5 .

$$
\text { Availability (1,2)=Availability (1) } \times \text { Availability (2) }
$$

Using the same tool, availability of each node for direct connection of Node 1 (Base Station) to other nodes in the network (the PMP Topology) and from the former to other nodes through intermediate nodes are as depicted in Table 6 below.

The implications of the above results obtained are that the combined availability of point to multipoint (PMP) components in series is always higher than the availability of its individual backhauling point to point (PTP) components. The maximum availability for the PMP 99.99, while for PTP is 98.99 .

\section{On throughput, latency and back to back tests}

Results obtained for the Throughput, Latency and Back to Back simulation tests on the test network also show that the PMP Topology is preferable to the PTP Topology.

Throughput test: The result Throughput test obtained for varying frame sizes (octets) for both the PTP and PMP Topologies are as depicted in Table 7.

From the Table and as depicted in Table 8, the PMP Topology affords better throughput (data rate) than the PTP Topology (Figure 13).

Latency test: For the latency test, similar results to the foregone were recorded. The result is as in Table 8 (Figure 14).

The results show reduced latency for each of the frame sizes for the PMP Topology compared to the PTP Topology.

Back to back test: Finally, on the network performance tests, the result for the Back to Back Simulation Test also vindicates PMP Topology as the preferred topology. Table below shows the results of the test for varying frame sizes (in octets) (Figure 15) (Table 9).

\begin{tabular}{|l|l|l|l|l|l|l|l|}
\hline $\begin{array}{l}\text { Availabilityl } \\
\text { Node }\end{array}$ & $\mathbf{1}$ & $\mathbf{2}$ & $\mathbf{3}$ & $\mathbf{4}$ & $\mathbf{5}$ & $\mathbf{6}$ & $\mathbf{7}$ \\
\hline $\mathbf{1}$ & ----- & 99.99 & 99.99 & 99.99 & 99.99 & 99.99 & 99.99 \\
\hline $\mathbf{2}$ & 98.99 & ----- & 97.5 & 98.99 & 97.5 & 97.5 & 99.99 \\
\hline $\mathbf{3}$ & 98.99 & 97.5 & ---- & 97.5 & 98.99 & 97.5 & 98.99 \\
\hline $\mathbf{4}$ & 98.99 & 97.5 & 97.5 & ----- & 96.55 & 97.5 & 97.5 \\
\hline $\mathbf{5}$ & 98.99 & 97.5 & 98.99 & 96.55 & ---- & 97.5 & 98.99 \\
\hline $\mathbf{6}$ & 98.99 & 97.5 & 97.5 & 97.5 & 97.5 & ---- & 98.99 \\
\hline $\mathbf{7}$ & 98.99 & 98.99 & 97.5 & 97.5 & 98.99 & 98.99 & ---- \\
\hline
\end{tabular}

Table 6: PTP/PMP availability chart.

Key: PTP Mode PMP Mode $\square$

\begin{tabular}{|l|l|l|l|l|l|l|l|}
\hline $\begin{array}{l}\text { Frame Size } \\
\text { (octets) }\end{array}$ & $\mathbf{6 4}$ & $\mathbf{1 2 8}$ & $\mathbf{2 5 6}$ & $\mathbf{5 1 2}$ & $\mathbf{1 0 2 4}$ & $\mathbf{1 2 8 0}$ & 1518 \\
\hline \multicolumn{8}{|c|}{ Maximum data rate (Mb/s) } \\
\hline PTP & 125 & 110 & 128 & 124 & 132 & 127 & 125 \\
\hline PMP & 130 & 115 & 135 & 130 & 139 & 133 & 139 \\
\hline
\end{tabular}

Table 7: PTP/PMP throughput results.

\begin{tabular}{|l|l|l|l|l|l|l|l|}
\hline Frame size(octets) & 64 & 128 & 256 & 512 & 1024 & 1280 & 1518 \\
\hline PTP Latency (uSecs) & 450 & 490 & 510 & 600 & 605 & 690 & 730 \\
\hline PMP Latency (uSecs) & 425 & 450 & 490 & 510 & 600 & 605 & 690 \\
\hline
\end{tabular}

Table 8: PTP/PMP latency test results.
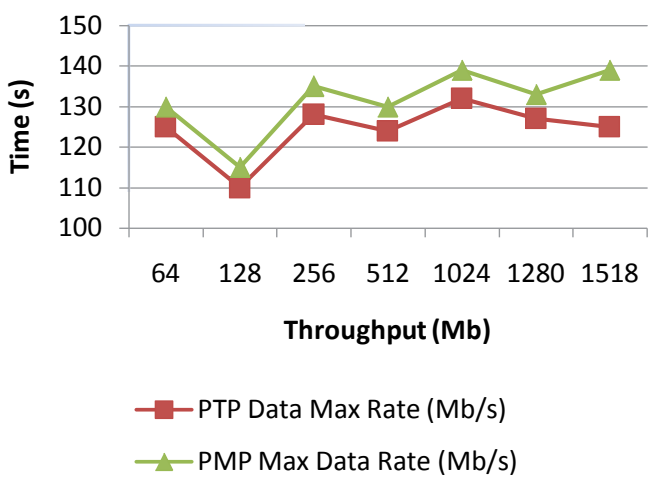

Figure 13: PTP/PMP throughput results.

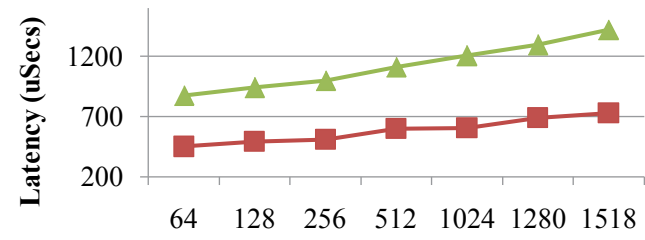

Frame Size (octets)

$\neg$ PTP Latency (uSecs) $\_$PMP Latency (uSecs)

Figure 14: PTP/PMP latency test results.

\begin{tabular}{|l|c|c|c|c|c|c|c|}
\hline $\begin{array}{l}\text { Frame size } \\
\text { (octets) }\end{array}$ & 64 & 128 & 256 & 512 & 1024 & 1280 & 1518 \\
\hline PTP (kb/s) & 3000 & 2500 & 3000 & 3250 & 3600 & 3100 & 3200 \\
\hline PMP (kb/s) & 3100 & 2600 & 3500 & 3450 & 3600 & 3400 & 3500 \\
\hline
\end{tabular}

Table 9: Back to back test results.

\begin{tabular}{|c|c|c|c|}
\hline Number of Nodes & $\begin{array}{c}\text { PMP Channel } \\
\text { Usage }\end{array}$ & $\begin{array}{c}\text { PTP Channel } \\
\text { Usage }\end{array}$ & $\begin{array}{c}\text { Relative Spectrum } \\
\text { Efficiency }\end{array}$ \\
\hline 1 & 1 & & \\
\hline 2 & 1 & 1 & 0.5 \\
\hline 3 & 1 & 2 & 0.33 \\
\hline 4 & 1 & 3 & 0.25 \\
\hline 5 & 1 & 4 & 0.20 \\
\hline 6 & 1 & 5 & 0.20 \\
\hline 7 & 1 & 5 & \\
\hline
\end{tabular}

Table 10: Relative spectrum efficiency for PTP/PMP.

\section{On spectrum utilization efficiency}

Using the relative spectral efficiency, RSE, defined under Section 3.1.9 and the deduction that the Spectral Efficiency is a function of radio frequency channel usage as depicted in the table for the test network under discuss, useful inferences can be made to compare the spectrum utilization over the PTP and PMP topologies (Table 10).

From Table 10 above, the relative spectrum efficiency for the PTP topology compared to that of the PMP Topology for varying number of nodes in the network is lower than for the PMP Topology. The advantage of the latter over the former in terms of spectrum utilization is thus demonstrated. This has direct bearing over operation expenditure (OPEX) and thus proves the PMP topology a better topology option. 
Citation: Ogunwolu L, Ibidapo-Obe O, Adeyemi T (2015) An Optimized Telecommunication System Topology for Expenditure Minimization and Improved Spectral Utilization Efficiency. J Telecommun Syst Manage 4: 119. doi:10.4172/2167-0919.1000119

Page 9 of 9

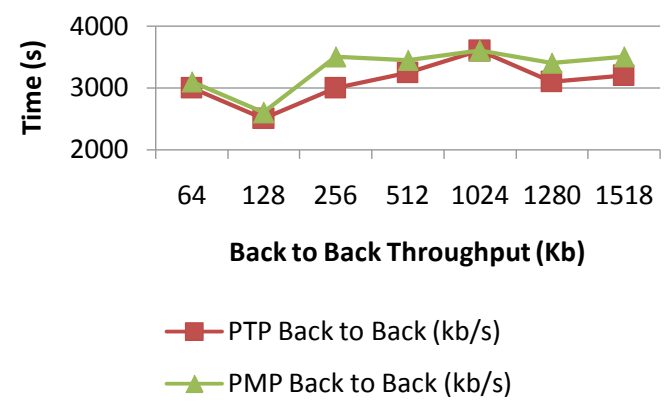

Figure 15: PTP/PMP back to back throughput test results.

\section{Conclusions and Extensions}

In this paper, the performance of wireless communication networks with significant reduction in hardware and signal processing costs is discussed on the bases of capital expenditure (CAPEX) influenced by network topology measures such as free path loss, availability, latency and channel frequency allocation, as well as operation expenditure (OPEX) influenced by network performance measures such as throughput, latency, back to back data rate and the expenditure intensive spectrum utilization efficiency.

The PMP topology was proved to outperform the PTP topology commonly in vogue. The effectiveness of Topology that makes use of point to multipoint method of backhauling was determined with our test network which allow the use of frequency division duplex (FDD) in the network of chosen topology.

Areas for future extensions include testing of the proposed PMP on backhauling in highly populated area to obtain experimental validation of the control methodology discussed.

There is still avenue for more research on optimization and spectral efficiency as numerous published works only focus on the cognitive radio with no emphasis on Topology, automatic gain control and weight sharing concept.

There is need to develop expert system that will take cognizance of the model described in this work and implement solution proposed in this project irrespective of the population of the end users as the model gives room for serial expansion.

The future research can also examine the importance of network topology to the time and frequency packing, FEC optimization, constant physical layer framing, pre-coding with "user-clustering", interference cancellation/multi-user detection at the receiver and higher order modulation schemes.

More studies should elaborate on roadmap of femto cells that will enhance the design of interference free of spectrum-shared miniaturized cell network.

More areas for future research investigation include information flow procedures, documented policies on efficient flow strategy, cognate analysis of utilization, the efficiency and radio standard optimization with the cognizance of interoperability and handover of heterogeneous network types.

\section{References}

1. Mitola J, Maguire GQ (1999) Cognitive radios: making software radios more personal. IEEE Personal Communications 6: 10-20.

2. http://telecom.esa.int/telecom/www/object/index.cfm?fobjectid=32272.

3. Kim H, Shin K (2008) Efficient discovery of spectrum opportunities with maclayer sensing in cognitive radio networks. Mobile Computing, IEEE Transactions 7: 533-545.

4. Quan Z, Cui S, Sayed AH, Vincent Poor H (2008) Wideband spectrum sensing in cognitive radio networks.

5. Suraweera HA, Gao J, Smith PJ, Shafi M, Faulkner M (2008) Channel capacity limits of cognitive radio in asymmetric fading environments.

6. Zhang R, Gao F, Liang YC (2009) Cognitive beam forming made practical Effective Interference Channel and Learning-Throughput Tradeoff.

7. Apurva K, Desai KR (2013) Design of clustered architecture for cooperative spectrum sensing with optimization of number of clusters. International Journal of Advances in Computer Science and Technology 2: 177-182.

8. Rashidi M, Haghighi K, Owrang A, Viberg M (2011) A wideband spectrum sensing method for cognitive radio using sub-Nyquist sampling. IEEE.

9. Singh SK, Roy KC, Pathak V (2010) Channels reallocation in cognitive radio networks based on DNA sequence alignment. International Journal of NextGeneration Networks 2: 23-34.

10. Taha Hamdy A (2011) Operations Research - An introduction (9thedn.) Pearson Education, New Jersey. 